\title{
The Total Quasi-Steady-State Approximation for Fully Competitive Enzyme Reactions
}

\author{
Morten Gram Pedersen * \\ Department of Mathematics, Technical University of Denmark, Kgs. Lyngby, Denmark
}

\author{
Alberto M. Bersani \\ Department of Mathematical Methods and Models, "La Sapienza” University, Rome, \\ Italy \\ Enrico Bersani
}

Datalink Informatica, Rome, Italy

\begin{abstract}
The validity of the Michaelis-Menten approximation for single enzyme reactions has recently been improved by the formalism of the total quasi-steady state assumption. This approach is here extended to fully competitive systems, and a criterion for its validity is provided. We show that it extends the Michaelis-Menten approximation for such systems for a wide range of parameters very convincingly, and investigate special cases. It is demonstrated that our method is at least roughly valid in the case of identical affinities. The results presented should be useful for numerical simulations of many in vivo reactions.
\end{abstract}

Key words: Michaelis-Menten kinetics, competitive substrates, substrate-inhibitor system, quasi steady-state assumption. 
* Corresponding author. Address: Department of Mathematics, Technical University of Denmark, Matematiktorvet, Building 303, DK-2800 Kgs. Lyngby, Denmark, Fax (+45) 45881399.

Email addresses: m.g.pedersen@mat.dtu.dk (Morten Gram Pedersen), bersani@dmmm . uniroma1.it (Alberto M. Bersani), e.bersani@datalinkinformatica.com(Enrico Bersani). 


\section{Introduction}

Biochemistry in general and enzyme kinetics in particular have been heavily influenced by the model of biochemical reactions set forth by Henri (1901a,b, 1902) and Michaelis and Menten (1913), and further developed by Briggs and Haldane (1925). This formulation considers a reaction where a substrate $S$ binds reversibly to an enzyme $E$ to form a complex $C$. The complex can decay irreversibly to a product $P$ and the enzyme, which is then free to bind another substrate molecule. This is summarized in the scheme

$$
E+S \stackrel{a}{\stackrel{a}{\rightleftarrows}} C \stackrel{k}{\longrightarrow} E+P
$$

where $a, d$ and $k$ are kinetic parameters (supposed constant) associated with the reaction rates.

Assuming that the complex concentration is approximately constant after a short transient phase leads to the usual Michaelis-Menten (MM) approximation (or standard quasi steady-state assumption or approximation (standard QSSA, sQSSA)), which is valid when the enzyme concentration is much lower than either the substrate concentration or the Michaelis-Menten constant $K_{M}$ (Segel, 1988). This is usually fulfilled for in vitro experiments, but often breaks down in vivo (Straus and Goldstein, 1943; Sols and Marco, 1970). See Schnell and Maini (2003) for a nice and complete review of the kinetics and approximations of scheme (1).

The advantage of a quasi steady-state approximation is that it reduces the dimensionality of the system, and thus speeds up numerical simulations greatly, especially for large networks as found in vivo. Moreover, while the kinetic constants in (1) are usually not known, finding the kinetic parameters characterizing the MM approximation is a standard procedure in in vitro biochemistry (Bisswanger, 2002). However, to simulate physiologically realistic in vivo scenarios, one faces the problem that the MM approximation is no longer valid as mentioned above. Hence, even 
though the kinetic constants such as $K_{M}$ are identical in vivo and in vitro, they need to be implemented in some other approximation which must be valid for the whole system and initial concentrations under investigation.

Approximations such as the reverse QSSA (rQSSA) (Segel and Slemrod, 1989; Schnell and Maini, 2000), which is valid for high enzyme concentrations, and the total QSSA (tQSSA) (Borghans et al., 1996; Tzafriri, 2003), which is valid for a broader range of parameters covering both high and low enzyme concentrations, have been introduced in the last two decades. Tzafriri (2003) showed that the tQSSA is at least roughly valid for any set of parameters. Also, the tQSSA for reversible reactions has been studied (Tzafriri and Edelman, 2004), i.e. reactions of form (1), but where enzyme and product can recombine to form the complex.

These newer approximations have so far only been found for isolated reactions. However, in vivo the reactions are coupled in complex networks or cascades of intermediate, second messengers with successive reactions, competition between substrates, feedback loops etc. Approximations of such scenarios have been carried out within the MM scheme (Bisswanger, 2002), but often without a thorough investigation of the validity of the approximations. An exception is the case of fully competitive reactions (Segel, 1988; Schnell and Mendoza, 2000), i.e., reactions with competing substrates, also known as substrate-inhibitor systems,

$$
\begin{aligned}
& S_{1}+E \underset{d_{1}}{\stackrel{a_{1}}{\rightleftarrows}} C_{1} \stackrel{k_{1}}{\longrightarrow} E+P_{1}, \\
& S_{2}+E \underset{d_{2}}{\stackrel{a_{2}}{\rightleftarrows}} C_{2} \stackrel{k_{2}}{\longrightarrow} E+P_{2},
\end{aligned}
$$

where $S_{i}, C_{i}$ and $P_{i}$ represent substrate, enzyme-substrate complex and product $i=1,2$, respectively. However, since the MM approximation cannot be expected to be valid in vivo, employing the tQSSA to these more complex situations would be beneficial.

This paper investigates the tQSSA for fully competitive reactions and is organized 
as follows. In Section 2 we recall the most important results in terms of quasi steady-state approximations for a single reaction and for a fully competitive system. In Section 3 we introduce the tQSSA for a fully competitive system, discussing the time scales of the reactions and introduce a sufficient condition for the validity of the tQSSA. Moreover, the form of the concentrations of the complexes $C_{i}$ in the quasi steady-state phase is investigated. In Section 4 we study the special case of identical affinities $\left(K_{1}^{M} \approx K_{2}^{M}\right)$. The first order approximation is obtained in terms of a perturbation parameter $r$, related to the characteristic constants of the systems. Finally, a closed form solution for the total substrate concentrations is obtained in this special case. In Section 5 the situation of very different affinities, for example reflecting a slow or fast inhibitor, is studied. The corresponding approximations for the concentrations of $C_{i}$ are found and used to obtain a general first order approximation to the tQSSA for fully competitive reactions for any choice of $K_{i}^{M}$, by means of Padé approximant techniques. In Section 6 we show numerically that for a very large range of parameters our tQSSA provides excellent fitting to the solutions of the full system, better than the sQSSA and the single reaction tQSSA, and we discuss the obtained results.

\section{Theoretical background}

We recall briefly the mathematical description of the sQSSA for (1), using the same symbols for the concentrations of the reactants. The reaction (1) can be described by the following system of nonlinear ordinary differential equations

$$
\begin{aligned}
& \frac{d S}{d t}=-a\left(E_{T}-C\right) S+d C \\
& \frac{d C}{d t}=a\left(E_{T}-C\right) S-(d+k) C
\end{aligned}
$$

with the initial conditions

$$
S(0)=S_{T}, \quad C(0)=0,
$$


and the conservation laws

$$
E+C=E_{T}, \quad S+C+P=S_{T} .
$$

Assuming that the complex is in a quasi-steady state leads to (Briggs and Haldane, 1925; Segel, 1988; Segel and Slemrod, 1989)

$$
\begin{gathered}
\frac{d S}{d t} \approx-\frac{V_{\max } S}{K_{M}+S}, \quad S(0)=S_{T}, \\
E(0)=E_{T}, \quad V_{\max }=k E_{T}, \quad K_{M}=\frac{d+k}{a} .
\end{gathered}
$$

Here $V_{\max }$ is the maximal reaction rate and $K_{M}$ is the Michaelis-Menten constant, identifying the substrate concentration giving the half-max reaction rate, i.e., $K_{M}$ reflects the substrate affinity of the enzyme. This approximation is valid whenever (Segel, 1988; Segel and Slemrod, 1989)

$$
\frac{E_{T}}{K_{M}+S_{T}} \ll 1,
$$

i.e., at low enzyme concentrations.

The tQSSA (Borghans et al., 1996; Tzafriri, 2003) arises by introducing the total substrate

$$
\bar{S}=S+C .
$$

Assuming that the complex is in a quasi-steady state yields the tQSSA

$$
\frac{d \bar{S}}{d t} \approx-k C_{-}(\bar{S}), \quad \bar{S}(0)=S_{T},
$$

where

$$
C_{-}(\bar{S})=\frac{\left(E_{T}+K_{M}+\bar{S}\right)-\sqrt{\left(E_{T}+K_{M}+\bar{S}\right)^{2}-4 E_{T} \bar{S}}}{2} .
$$

Tzafriri (2003) showed that the tQSSA is valid whenever

$$
\epsilon_{T z}:=\frac{K}{2 S_{T}}\left(\frac{E_{T}+K_{M}+S_{T}}{\sqrt{\left(E_{T}+K_{M}+S_{T}\right)^{2}-4 E_{T} S_{T}}}-1\right) \ll 1, \quad K=\frac{k}{a},
$$


and that this is always roughly valid in the sense that

$$
\epsilon_{T z} \leq \frac{K}{4 K_{M}} \leq \frac{1}{4}
$$

The parameter $K$ is known as the Van Slyke-Cullen constant. Tzafriri (2003) expanded equations (10) and (11) in terms of

$$
r(\bar{S})=\frac{4 E_{T} \bar{S}}{\left(E_{T}+K_{M}+\bar{S}\right)^{2}}<1
$$

and assuming the validity of the tQSSA $\left(\epsilon_{T z} \ll 1\right)$ and $r \ll 1$, he found

$$
\frac{d \bar{S}}{d t} \approx-\frac{V_{\max } \bar{S}}{K_{M}+E_{T}+\bar{S}}, \quad \bar{S}(0)=S_{T}
$$

as a first order approximation to (9). This expression (14) is identical to the formula obtained by Borghans et al. (1996) by means of a two point Padé approximant technique (Baker, 1975).

This approximation is valid at low enzyme concentrations (8) where it reduces to the MM expression (7), but holds moreover at low substrate concentrations $S_{T} \ll E_{T}+K_{M}$ (Tzafriri, 2003). We wish to highlight the fundamental fact that performing the substitutions of $S$ by $\bar{S}$ and of $K_{M}$ by $K_{M}+E_{T}$ one obtains a significantly improved MM-like approximation with minimal effort.

The system (2) under investigation in this paper is governed by the coupled ODEs (Rubinow and Lebowitz, 1970; Segel, 1988; Schnell and Mendoza, 2000), $i=1,2$,

$$
\begin{array}{rlrl}
\frac{d S_{i}}{d t} & =-a_{i} E \cdot S_{i}+d_{i} C_{i}, & & S_{i}(0)=S_{i, T}, \\
\frac{d C_{i}}{d t}=a_{i}\left(E \cdot S_{i}-K_{i}^{M} C_{i}\right), & C_{i}(0)=0, & K_{i}^{M}=\frac{d_{i}+k_{i}}{a_{i}} .
\end{array}
$$

and the conservation laws

$$
\begin{aligned}
S_{i, T} & =S_{i}+C_{i}+P_{i}, \quad i=1,2, \\
E_{T} & =E+C_{1}+C_{2} .
\end{aligned}
$$


The sQSSA of this system is (Rubinow and Lebowitz, 1970; Segel, 1988)

$$
\frac{d S_{i}}{d t}=-\frac{k_{i} E_{T} S_{i}}{K_{i}^{M}\left(1+S_{j} / K_{j}^{M}\right)+S_{i}}, \quad S_{i}(0)=S_{i, T}, \quad i=1,2, \quad j \neq i,
$$

which is valid when (Schnell and Mendoza, 2000)

$$
\frac{E_{T}}{K_{i}^{M}\left(1+S_{j, T} / K_{j}^{M}\right)+S_{i, T}} \ll 1, \quad i=1,2, \quad j \neq i .
$$

\section{Total quasi-steady state approximation of the competitive system}

Following Borghans et al. (1996), we introduce the total substrates

$$
\bar{S}_{i}=S_{i}+C_{i}, \quad i=1,2,
$$

and rewrite equations (15) in terms of these, obtaining the system of ODEs, $i=1,2$,

$$
\begin{array}{ll}
\frac{d \bar{S}_{i}}{d t}=-k_{i} C_{i}, & \bar{S}_{i}(0)=S_{i, T} \\
\frac{d C_{i}}{d t}=a_{i}\left(\left(E_{T}-C_{1}-C_{2}\right) \cdot\left(\bar{S}_{i}-C_{i}\right)-K_{i}^{M} C_{i}\right), & C_{i}(0)=0 .
\end{array}
$$

We require $0<C_{i}<\bar{S}_{i}, i=1,2$, because of (20), and apply the quasi steady-state assumption (Borghans et al., 1996; Tzafriri, 2003),

$$
\frac{d C_{i}}{d t} \approx 0, \quad i=1,2
$$

which is equivalent to the system

$$
\begin{aligned}
& C_{1}=E_{T}-C_{2}\left(1+\frac{K_{2}^{M}}{\bar{S}_{2}-C_{2}}\right), \\
& C_{2}=E_{T}-C_{1}\left(1+\frac{K_{1}^{M}}{\bar{S}_{1}-C_{1}}\right) .
\end{aligned}
$$

Then $C_{i}<E_{T}, i=1,2$, in agreement with (17). We now show the existence and uniqueness of a solution to the system (22) with $0<C_{i}<\min \left\{\bar{S}_{i}, E_{T}\right\}$. 
First we note that (22) implies

$$
\frac{K_{1}^{M} C_{1}}{\bar{S}_{1}-C_{1}}=\frac{K_{2}^{M} C_{2}}{\bar{S}_{2}-C_{2}}
$$

from which it is seen that $0<C_{1}<\bar{S}_{1}$ if and only if $0<C_{2}<\bar{S}_{2}$.

Substituting (22b) into (22a) leads to the following equation in $C_{1}$

$$
C_{1}=E_{T}-\left(E_{T}-C_{1}\left(1+\frac{K_{1}^{M}}{\bar{S}_{1}-C_{1}}\right)\right)\left(1+\frac{K_{2}^{M}}{\bar{S}_{2}-\left(E_{T}-C_{1}\left(1+\frac{K_{1}^{M}}{S_{1}-C_{1}}\right)\right)}\right)
$$

and $C_{2}$ can then be found from (22b).

Solving (23) is equivalent to finding roots of the third degree polynomial

$$
\begin{aligned}
\psi_{1}\left(C_{1}\right) & =-\left(K_{1}^{M}-K_{2}^{M}\right) C_{1}^{3} \\
& +\left[\left(E_{T}+K_{1}^{M}+\bar{S}_{1}\right)\left(K_{1}^{M}-K_{2}^{M}\right)-\left(\bar{S}_{1} K_{2}^{M}+\bar{S}_{2} K_{1}^{M}\right)\right] C_{1}^{2} \\
& +\left[-E_{T}\left(K_{1}^{M}-K_{2}^{M}\right)+\left(\bar{S}_{1} K_{2}^{M}+\bar{S}_{2} K_{1}^{M}\right)+K_{2}^{M}\left(E_{T}+K_{1}^{M}\right)\right] \bar{S}_{1} C_{1} \\
& -E_{T} K_{2}^{M} \bar{S}_{1}^{2} .
\end{aligned}
$$

An analogous polynomial $\psi_{2}$ for $C_{2}$ can be found by interchanging the indexes 1 and 2 in (24), because of the symmetry of the system (21). Rearranging the terms, $\psi_{1}$ can also be written

$$
\begin{aligned}
\psi_{1}\left(C_{1}\right)= & K_{2}^{M}\left(C_{1}-E_{T}\right)\left(\bar{S}_{1}-C_{1}\right)^{2} \\
& \left.+K_{1}^{M} C_{1}\left(C_{1}+K_{2}^{M}+\bar{S}_{2}-E_{T}\right)\right)\left(\bar{S}_{1}-C_{1}\right)+\left(K_{1}^{M} C_{1}\right)^{2}
\end{aligned}
$$

From (24) we see that $\psi_{1}(0)<0$, and from $(25)$ that $\psi_{1}\left(\bar{S}_{1}\right)>0$. Hence, $\psi_{1}$ has at least one root between 0 and $\bar{S}_{1}$, which shows existence.

When $K_{1}^{M} \neq K_{2}^{M}$, we can without loss of generality assume that $K_{1}^{M}>K_{2}^{M}$ because of the symmetry of (2). In this case $\lim _{c \rightarrow \pm \infty} \psi_{1}(c)=\mp \infty$, and we see that $\psi_{1}$ has one negative root and one root larger than $\bar{S}_{1}$. Hence, there is a unique root $C_{1} \in\left(0, \bar{S}_{1}\right)$, which also solves (23). This implies the uniqueness of the solution. When $K_{1}^{M}=K_{2}^{M}=K_{M}, \psi_{1}$ becomes a second degree polynomial. Because of (24) we have $\lim _{c \rightarrow \infty} \psi_{1}(c)=-\infty$, so the second root is larger than $\bar{S}_{1}$. Hence, also in 
this case we have only one root between 0 and $\bar{S}_{1}$, given by

$$
C_{1}=\frac{\bar{S}_{1}\left(\bar{S}_{1}+\bar{S}_{2}+K_{M}+E_{T}\right)}{2\left(\bar{S}_{1}+\bar{S}_{2}\right)}\left(1-\sqrt{1-\frac{4 E_{T}\left(\bar{S}_{1}+\bar{S}_{2}\right)}{\left(\bar{S}_{1}+\bar{S}_{2}+K_{M}+E_{T}\right)^{2}}}\right) .
$$

The approach to solving (22) taken above helps the theoretical reasoning, but is practically cumbersome, since we need to find the largest $K_{i}^{M}$. In addition, the formula (22b) for finding $C_{2}$ is numerically imprecise when both $C_{1}$ and $\bar{S}_{1}$ are small. Both these problems can be overcome by finding the root of the polynomial $\psi_{2}$ for $C_{2} ; \psi_{2}$ has a single root in $\left(0, \bar{S}_{2}\right)$ as a consequence of the uniqueness result.

\subsection{Validity of the tQSSA}

We expect that after a short transient phase the complex concentrations equal the quasi steady-state concentrations, $C_{i}=C_{i}\left(\bar{S}_{1}, \bar{S}_{2}\right)$, given by the roots in the respective polynomials as discussed above. Then the evolution of the system can be studied by means of the tQSSA

$$
\frac{d \bar{S}_{i}}{d t} \approx-k_{i} C_{i}\left(\bar{S}_{1}, \bar{S}_{2}\right), \quad \bar{S}_{i}(0)=S_{i, T}
$$

Segel (1988) proposed the following two criteria for the validity of a QSSA.

(i) The time scale for the complex(es) during the transient phase, $t_{C}$, should be much smaller than the time scale for changes in the substrate(s) in the beginning of the quasi steady-state phase, $t_{S}$.

(ii) The substrate(s) should be nearly constant during the transient phase.

In our case, (ii) can be translated to (Segel, 1988; Tzafriri, 2003)

$$
\frac{S_{i, T}-\bar{S}_{i}}{S_{i, T}} \leq \frac{t_{C}}{S_{i, T}} \max \left|\frac{d \bar{S}_{i}}{d t}\right|=\frac{k_{i} t_{C}}{S_{i, T}} C_{i}\left(S_{1, T}, S_{2, T}\right) \ll 1, \quad i=1,2,
$$

where the maximum is taken over the transient phase, i.e., with $\bar{S}_{i} \approx S_{i, T}$. Since $C_{i}$ is increasing during the transient phase, the maximum is given by $k_{i} C_{i}\left(S_{1, T}, S_{2, T}\right)$. 
The substrate time scale (Segel, 1988; Tzafriri, 2003) is estimated from (27) to be

$$
t_{\bar{S}_{i}} \approx \frac{S_{i, T}}{k_{i} C_{i}\left(S_{1, T}, S_{2, T}\right)}
$$

and we see that (28) translates into (i), i.e.,

$$
\max _{i=1,2} \frac{t_{C}}{t_{\bar{S}_{i}}}=\frac{\max \left\{t_{C_{1}}, t_{C_{2}}\right\}}{\min \left\{t_{\bar{S}_{1}}, t_{\bar{S}_{2}}\right\}} \ll 1
$$

The time scale for the complexes is estimated following Borghans et al. (1996):

$$
t_{C_{i}} \approx \frac{C_{i}\left(S_{1, T}, S_{2, T}\right)}{\max \left|\frac{d C_{i}}{d t}\right|}=\frac{C_{i}\left(S_{1, T}, S_{2, T}\right)}{a_{i} E_{T} S_{i, T}},
$$

where the maximum is again taken during the transient phase. The time scale for the transient phase is then the maximum of the two individual scales; we want both complexes to be near the equilibrium at the end of the transient phase, and both substrates to be nearly constant during it.

Hence, we propose the following sufficient condition for the validity of our tQSSA (27),

$$
\epsilon:=\max _{i=1,2}\left(\frac{k_{i} C_{i}\left(S_{1, T}, S_{2, T}\right)}{S_{i, T}}\right) \max _{i=1,2}\left(\frac{C_{i}\left(S_{1, T}, S_{2, T}\right)}{a_{i} E_{T} S_{i, T}}\right) \ll 1 .
$$

Whenever the two maxima occur for the same $i$, (32) simplifies to

$$
\bar{\epsilon}=\max _{i=1,2}\left(\frac{K_{i}}{E_{T}}\left(\frac{C_{i}\left(S_{1, T}, S_{2, T}\right)}{S_{i, T}}\right)^{2}\right) \ll 1,
$$

where we introduced the Van Slyke-Cullen constants $K_{i}=k_{i} / a_{i}$.

\section{Identical affinity, $K_{1}^{M} \approx K_{2}^{M}$.}

When $K_{1}^{M} \neq K_{2}^{M}$ the roots of $\psi_{1}$ are given by a very complicated formula in contrast to the formula (26) when $K_{1}^{M} \approx K_{2}^{M}=K_{M}$. To deepen our understanding of the problem we follow this latter case further. It should be noted that the situation is biologically realistic, for example for bacterial chitooligosaccharide with chitotriose and chitopentose as competitive substrates (Pi and Leary, 2004), for 
$\mathrm{I} \kappa \mathrm{B}$ kinase (IKK-2) phosphorylation of $\mathrm{I} \kappa \mathrm{B} \alpha$ and $\mathrm{p} 65$, which is of importance in inflammatory diseases (Kishore et al., 2003) and for the double phosphorylation of MAPK by MAPKK (Huang and Ferrell, 1996; Bhalla and Iyengar, 1999; Kholodenko, 2000). Following Tzafriri we let

$$
r(\bar{S})=\frac{4 E_{T} \bar{S}}{\left(\bar{S}+K_{M}+E_{T}\right)^{2}} .
$$

Then we can rewrite (26) as

$$
C_{1}\left(\bar{S}_{1}, \bar{S}_{2}\right)=\frac{\bar{S}_{1}\left(\bar{S}_{1}+\bar{S}_{2}+K_{M}+E_{T}\right)}{2\left(\bar{S}_{1}+\bar{S}_{2}\right)}\left(1-\sqrt{1-r\left(\bar{S}_{1}+\bar{S}_{2}\right)}\right)
$$

Setting

$$
K=\frac{\max \left\{k_{1}, k_{2}\right\}}{\min \left\{a_{1}, a_{2}\right\}}, \quad S_{T}=S_{1, T}+S_{2, T}, \quad r_{T}=r\left(S_{T}\right),
$$

we get from (32) and (34) that

$$
\begin{aligned}
\epsilon & =\frac{K}{E_{T}}\left(\frac{S_{T}+K_{M}+E_{T}}{2 S_{T}}\left(1-\sqrt{1-r_{T}}\right)\right)^{2} \\
& =\frac{K}{S_{T}} \frac{\left(1-\sqrt{1-r_{T}}\right)^{2}}{1-\left(1-r_{T}\right)} \\
& =\frac{K}{S_{T}} \frac{1-\sqrt{1-r_{T}}}{2} \frac{2}{1+\sqrt{1-r_{T}}} \\
& \leq \frac{K}{2 S_{T}} \frac{1-\sqrt{1-r_{T}}}{\sqrt{1-r_{T}}} \\
& =\epsilon_{T z}
\end{aligned}
$$

where $\epsilon_{T z}$ is the expression from (11). Let us remark that the Van Slyke - Cullen constant $K$ in (35) is different from the corresponding constant appearing in a single reaction. Assuming $K \leq K_{M}$, we can use the result (12) to get

$$
\epsilon \leq \epsilon_{T z} \leq \frac{K}{4 K_{M}} \leq \frac{1}{4}
$$

This is fulfilled if $K=k_{i} / a_{i}$ (same $i$ ), but is not necessarily true if $K=k_{i} / a_{j}$ $(j \neq i)$. Inequality (36) tells us that, for identical affinities and under the assumption $K \leq K_{M}$, the tQSSA is at least roughly valid, and the smaller the ratio $K / K_{M}$, the better the approximation. 


\subsection{First order tQSSA for identical affinities}

Developing (34) in $r$ yields

$$
C_{1}=\frac{E_{T} \bar{S}_{1}}{\bar{S}_{1}+\bar{S}_{2}+K_{M}+E_{T}}+O\left(r^{2}\right)
$$

In this case (compare with Borghans et al. (1996))

$$
\epsilon=\frac{K E_{T}}{\left(S_{1, T}+S_{2, T}+K_{M}+E_{T}\right)^{2}}+O\left(r^{2}\right), \quad K=\frac{\max \left\{k_{1}, k_{2}\right\}}{\min \left\{a_{1}, a_{2}\right\}} .
$$

When $r \ll 1$ and the tQSSA is valid $(\epsilon \ll 1)$, we obtain the first order tQSSA (with respect to $r$ ) for competing substrates with identical affinity

$$
\frac{d \bar{S}_{i}}{d t} \approx-\frac{k_{i} E_{T} \bar{S}_{i}}{\bar{S}_{1}+\bar{S}_{2}+K_{M}+E_{T}}, \quad \bar{S}_{i}(0)=S_{i, T}, \quad i=1,2
$$

The sufficient conditions for $r \ll 1$ from Tzafriri (2003) translate into either of

$$
\begin{aligned}
& S_{1, T}+S_{2, T}+K_{M} \gg E_{T}, \\
& E_{T}+K_{M} \gg S_{1, T}+S_{2, T} .
\end{aligned}
$$

The condition (40) also guarantees $\epsilon \ll 1$ because of (38), unless $K \gg K_{M}+$ $S_{1, T}+S_{2, T}$. As noted above, $K \leq K_{M}$ if $K=k_{i} / a_{i}$ (same $i$ ), and then indeed $\epsilon \ll 1$.

However, (41) does not imply $\epsilon \ll 1$ but must be accompanied by $K \ll K_{M}$, in which case (36) guarantees $\epsilon \ll 0.25$. When $K \gtrsim K_{M}$ we must require $E_{T} \gg K$ such that (38) yields $\epsilon \ll 1$, and in this case (41) simplifies to $E_{T} \gg S_{1, T}+S_{2, T}$. In summary, any of the following conditions imply the validity of the first order tQSSA for identical affinities:

$$
\begin{array}{rll}
E_{T} \ll S_{1, T}+S_{2, T}+K_{M}, & \text { and } & K \gtrsim K_{M}+S_{1, T}+S_{2, T} \\
E_{T}+K_{M} \gg S_{1, T}+S_{2, T}, & \text { and } & K \ll K_{M}, \\
E_{T} \gg S_{1, T}+S_{2, T}, & \text { and } & E_{T} \gg K \gtrsim K_{M} .
\end{array}
$$


Neglecting $E_{T}$ in the denominator in (39) we obtain again the sQSSA of competing substrates with identical affinities. This is valid when (42) holds, as seen from (19). On the other hand, when (43) or (44) is fulfilled, (39) does not reduce to the sQSSA. Hence, (43) or (44) extend the parameter region where (39) is valid.

\subsection{Uncoupled equations and closed form solutions}

When $K_{1}^{M} \approx K_{2}^{M}$ as above, the two equations given by (27) can be uncoupled (Rubinow and Lebowitz, 1970; Segel, 1988; Schnell and Mendoza, 2000) by dividing one by the other and using (26), leading to

$$
\frac{d \bar{S}_{1}}{d \bar{S}_{2}}=\frac{k_{1}}{k_{2}} \frac{\bar{S}_{1}}{\bar{S}_{2}}
$$

such that

$$
\frac{\bar{S}_{1}}{S_{1, T}}=\left(\frac{\bar{S}_{2}}{S_{2, T}}\right)^{\delta}, \quad \delta=\frac{k_{1}}{k_{2}} .
$$

This relation can then be used to eliminate $\bar{S}_{2}$ in (26) and, similarly, eliminate $\bar{S}_{1}$ in the expression for $C_{2}$. It also shows that when $\delta=1$, i.e. $k_{1}=k_{2}=k_{c a t}$, the two substrates behave identically with the only difference given by their initial concentrations. This can also be observed from (27) with (26) inserted.

In the following we assume that the first order tQSSA holds. Using (45) we write (39) as

$$
\begin{array}{ll}
\frac{d \bar{S}_{1}}{d t} \approx-\frac{k_{1} E_{T} \bar{S}_{1}}{\bar{S}_{1}+S_{2, T}\left(\bar{S}_{1} / S_{1, T}\right)^{1 / \delta}+K_{M}+E_{T}}, & \bar{S}_{1}(0)=S_{1, T}, \\
\frac{d \bar{S}_{2}}{d t} \approx-\frac{k_{2} E_{T} \bar{S}_{2}}{\bar{S}_{2}+S_{1, T}\left(\bar{S}_{2} / S_{2, T}\right)^{\delta}+K_{M}+E_{T}}, & \bar{S}_{2}(0)=S_{2, T}
\end{array}
$$

These equations are identical to the ones studied by Schnell and Mendoza (2000) setting $K_{1}^{M}=K_{2}^{M}$ and applying the substitution $K_{M} \rightarrow K_{M}+E_{T}$. Hence, the same techniques can be used to find closed form solutions.

When $\delta=1,(46)$ and (47) are identical except from the initial conditions and hence the two substrates develop identically, as observed above. The solution is given in 
closed form by

$$
\bar{S}_{i}(t) \approx S_{i, T} \frac{K_{M}+E_{T}}{S_{1, T}+S_{2, T}} \cdot W\left(\frac{S_{1, T}+S_{2, T}}{K_{M}+E_{T}} \exp \left(\frac{S_{1, T}+S_{2, T}-k_{c a t} E_{T} t}{K_{M}+E_{T}}\right)\right),
$$

where $W$ is the Lambert $W$-function introduced in enzyme kinetics by Schnell and Mendoza (1997). It is defined as the real valued solution to

$$
W(x) \cdot \exp (W(x))=x
$$

At high enzyme concentrations (see formula (41)), the argument of $W$ in (48) is small and the approximation $W(x) \approx x$ holds. Hence

$$
\bar{S}_{i}(t) \approx S_{i, T} \exp \left(-\frac{k_{c a t} E_{T}}{K_{M}+E_{T}} t\right)
$$

which is identical to the expression for the tQSSA of an isolated reaction (Tzafriri, 2003). Hence, the two substrates behave completely independently as if they were isolated. The same result is found directly by neglecting $\bar{S}_{1}+\bar{S}_{2}$ in the denominator of (39). The biophysical reason is that the enzyme excess is so large that the substrate does not "sense" that some fraction of the enzyme is bound by the other substrate.

The case when $\delta \ll 1$ corresponds to a slow (resp. fast) inhibitor, when $\bar{S}_{1}$ is regarded as the inhibitor (resp. substrate) and $\bar{S}_{2}$ as the substrate (resp. inhibitor). The closed form solution can again be found following Schnell and Mendoza (2000), giving

$$
\begin{aligned}
& \bar{S}_{2}(t) \approx\left(S_{1, T}+K_{M}+E_{T}\right) W\left(\frac{S_{2, T}}{S_{1, T}+K_{M}+E_{T}} \exp \left(\frac{S_{2, T}-k_{2} E_{T} t}{S_{1, T}+K_{M}+E_{T}}\right)\right) \\
& \bar{S}_{1}(t) \approx S_{1, T}\left(\frac{\bar{S}_{2}(t)}{S_{2, T}}\right)^{\delta} .
\end{aligned}
$$




\section{$5 \quad K_{1}^{M} \gg K_{2}^{M}$ and the general first order approximation}

We now turn to the case of very different affinities as stated by $K_{1}^{M} \gg K_{2}^{M}$. To investigate this situation closer we perform a perturbation around $K_{2}^{M}=0$. When $K_{2}^{M}=0$, we see from (21b) that in the quasi-steady state, $C_{2} \approx \bar{S}_{2}$ or $E_{T}-C_{1}-C_{2} \approx 0$. In the former case, $C_{2} \approx S_{2, T}$ since $k_{2}=0$. In the latter case, again from (21b), but now for $i=1$, it follows that $C_{1} \approx 0, C_{2} \approx E_{T}$. Since $C_{2} \leq \min \left\{S_{2, T}, E_{T}\right\}$ we get that, when $K_{2}^{M}=0$,

$$
C_{2} \approx \min \left\{S_{2, T}, E_{T}\right\}
$$

We study these two cases independently, and by means of their corresponding solutions we build a two point Padé approximant (TPPA) (Baker, 1975) developed around $\eta=0\left(E_{T} \gg S_{2, T}\right)$ and $\eta=\infty\left(E_{T} \ll S_{2, T}\right)$.

When $E_{T} \gg S_{2, T}$ (i.e., $\eta \ll 1$ ), we expect that the system evolves as two independent reactions: $S_{2}$ binds rapidly with a part of the enzyme leaving $E_{T}^{*}=E_{T}-S_{2, T}$ to react with $S_{1}$. Hence, we obtain from (9) and (14)

$$
\begin{aligned}
\frac{d \bar{S}_{1}}{d t} & \approx-k_{1} \frac{\left(E_{T}^{*}+K_{1}^{M}+\bar{S}_{1}\right)-\sqrt{\left(E_{T}^{*}+K_{1}^{M}+\bar{S}_{1}\right)^{2}-4 E_{T}^{*} \bar{S}_{1}}}{2} \\
& \approx-\frac{k_{1} E_{T}^{*} \bar{S}_{1}}{K_{1}^{M}+E_{T}^{*}+\bar{S}_{1}} .
\end{aligned}
$$

Note that here $K_{2}^{M}=0$, such that (22a) and hence (23) are not valid. The solution can also be found by setting $C_{2}=S_{2, T}$ in (21b) with $i=1$.

In the case when $0<K_{2}^{M} \ll K_{1}^{M}$, we neglect terms involving $K_{2}^{M}$ in (24) and obtain that $C_{1}$ should satisfy

$$
\left(C_{1}^{2}-\left(E_{T}+K_{1}^{M}+\bar{S}_{1}-\bar{S}_{2}\right) C_{1}+\left(E_{T}-\bar{S}_{2}\right) \bar{S}_{1}\right) \cdot C_{1}=0 .
$$

Since $C_{2} \leq S_{2, T}<E_{T}, C_{1}=0$ is in contradiction with (22b). Thus, $C_{1}$ solves the second degree polynomial which is exactly the polynomial given from the tQSSA for an isolated reaction, i.e., $\bar{S}_{1}$ follows again (50) but now with $E_{T}^{*}=E_{T}-\bar{S}_{2}$. 
We now turn to the case when $C_{2} \approx E_{T} \ll S_{2, T}$ (i.e., $\eta \gg 1$ ). Recall that this is the case of the usual in vitro experiments. From the conservation law (17) $C_{1} \approx 0$. We expand $\psi_{1} / K_{1}^{M}$ in terms of the small parameter $\rho=K_{2}^{M} / K_{1}^{M}$ and find that the first order term for the root is given by

$$
C_{1}=\rho \frac{E_{T} \bar{S}_{1}}{\bar{S}_{2}-E_{T}}=\frac{K_{2}^{M}}{K_{1}^{M}} \times \frac{E_{T} \bar{S}_{1}}{\bar{S}_{2}-E_{T}} .
$$

Using (52) for $1 / \eta \approx 0$, and the first order approximation (51) for $\eta \approx 0$, the TPPA in $\eta=\bar{S}_{2} / E_{T}$ is

$$
C_{1}=\frac{E_{T} \bar{S}_{1}}{K_{1}^{M}+\bar{S}_{1}+E_{T}+\bar{S}_{2} / \rho}=\frac{E_{T} \bar{S}_{1}}{K_{1}^{M}\left(1+\bar{S}_{2} / K_{2}^{M}\right)+\bar{S}_{1}+E_{T}} .
$$

Plugging (53) into (27) (for $i=1$ ) yields then

$$
\frac{d \bar{S}_{i}}{d t}=-\frac{k_{i} E_{T} \bar{S}_{i}}{K_{i}^{M}\left(1+\bar{S}_{j} / K_{j}^{M}\right)+\bar{S}_{i}+E_{T}}, \quad \bar{S}_{i}(0)=S_{i, T}, \quad j \neq i .
$$

where $i=1, j=2$. Similar computations can be performed for $C_{2}$, when $K_{1}^{M} \ll$ $K_{2}^{M}$, yielding the same equation (52), where $i=2, j=1$.

The two approximations hold for two different regions of parameter space, $K_{1}^{M} \gg$ $K_{2}^{M}$ and $K_{1}^{M} \ll K_{2}^{M}$, respectively. However, let us observe that they reduce not only to the case of identical affinities, (39), for $K_{1}^{M}=K_{2}^{M}$, but also to the sQSSA (18) whenever this approximation holds as guaranteed by (19), and to the single reaction first order tQSSA (14) when $\bar{S}_{j} / K_{j}^{M}$ can be neglected.

Motivated by this and further encouraged by numerical simulations (see the following section), we propose the expression (54) (for $i=1,2$ ) as the general first order approximation to the tQSSA for fully competitive reactions.

Although not strictly theoretically founded, the above considerations using the TPPA can be seen as the motivation for the formula. However, as shown in the Appendix, we can indeed expect $C_{1}$ from (53) (and $C_{2}$ in the corresponding expression) to be a good approximation to the true root of $\psi_{1}\left(\psi_{2}\right.$ for $\left.C_{2}\right)$ when either 
(19) holds, or when

$$
K_{i}^{M} \gg S_{1, T}+S_{2, T} \quad \text { or } \quad E_{T} \gg K_{i}^{M}\left(1+S_{j, T} / K_{j}^{M}\right)+S_{i, T}, \quad i=1,2, j \neq i .
$$

Hence, (54) extends both the sQSSA (18) as well as the single reaction tQSSA.

The considerations in the Appendix tell us only when the first order tQSSA is a good approximation of the full tQSSA, but neither might be a good representation of the full system. To assure that, $\epsilon$ must be small.

When (53) approximates the full tQSSA we have from (32)

$$
\begin{aligned}
\epsilon & =\max _{i=1,2} \frac{k_{i} E_{T}}{\tilde{K}_{i, T}^{M}+S_{i, T}+E_{T}} \max _{i=1,2} \frac{1}{a_{i}\left(\tilde{K}_{i, T}^{M}+S_{i, T}+E_{T}\right)} \\
& \leq K \max _{i=1,2} \frac{E_{T}}{\tilde{K}_{i, T}^{M}+S_{i, T}+E_{T}} \max _{i=1,2} \frac{1}{\tilde{K}_{i, T}^{M}+S_{i, T}+E_{T}} \\
& =\max _{i=1,2} \frac{K E_{T}}{\left(\tilde{K}_{i, T}^{M}+S_{i, T}+E_{T}\right)^{2}}
\end{aligned}
$$

where

$$
K=\frac{\max \left\{k_{1}, k_{2}\right\}}{\min \left\{a_{1}, a_{2}\right\}}, \quad \tilde{K}_{i, T}^{M}=K_{i}^{M}\left(1+S_{j, T} / K_{j}^{M}\right), \quad i=1,2, j \neq i
$$

The above considerations yield that either one of the following conditions guarantees the validity of the first order approximation $(54)(i=1,2)$ :

$$
\begin{array}{rll}
E_{T} \ll S_{i, T}+\tilde{K}_{i, T}^{M}, & \text { and } & K \gtrsim \tilde{K}_{i, T}^{M}+S_{i, T}, \\
K_{i}^{M} \gg S_{1, T}+S_{2, T}, & \text { and } & K \ll \tilde{K}_{i, T}^{M}, \\
K_{i}^{M} \gg S_{1, T}+S_{2, T}, & \text { and } & E_{T} \gg K \gtrsim \tilde{K}_{i, T}^{M}, \\
E_{T} \gg \tilde{K}_{i, T}^{M}+S_{i, T}, & \text { and } & E_{T} \gg K .
\end{array}
$$

\section{Numerical Results and Discussion}

We have extended the total quasi-steady state assumption to competing substrates, investigating its validity and deepening some special cases. As seen in Fig. 1, the 
approximation is indeed excellent as long as $\epsilon$ is small. We have not found any values of the parameters for which numerical simulations show that our tQSSA breaks down dramatically. Of importance, our approximation captures the competition as does the sQSSA (18) and in contrast with the single reaction tQSSA (9), but also at intermediate or high enzyme concentrations where the sQSSA does not hold anymore (Fig. 1). However, when the competition can be neglected due to, e.g., low substrate concentrations, the single reaction tQSSA does indeed estimate the full system well (see, e.g., Fig. 2).

A crucial step of our analysis is finding the roots of the third degree polynomials $\psi_{i}$. Although we have shown that there is exactly one physically possible root for each complex, and that there exists, e.g., Cardano's formula for this root, the formula is hard to interpret and even to implement. We have used a differential-algebraic equations (DAE) approach, i.e., finding the roots numerically. Such a DAE approach is easier to implement than using the closed form for the root, but increases the time needed for computations.

These problems can partly be resolved by using approximations of the roots of $\psi_{i}$. Compared to the full solution, such an approximation should preferably be easier to interpret and to relate to previously known formulas. Furthermore, it should be clearly stated when it is valid. We found a first order approximation, which is valid when the MM approach is $((57))$, and in this case they coincide (Fig. 2A). Moreover, it is valid for high $K_{i}^{M}$ values (conditions (58) and (59)), where it reduces to the single reaction first order approximation (Fig. 2B). Hence, it extends these two approximations beyond the regions where they are known to hold. Finally, the first order approximation is valid at high enzyme concentrations (60), but it is not always accurate if the enzyme concentration is only moderately high. In this case, the single reaction tQSSA is often a better approximation (Fig. 2C).

In the special case of identical affinities we saw that our approach should be at least 


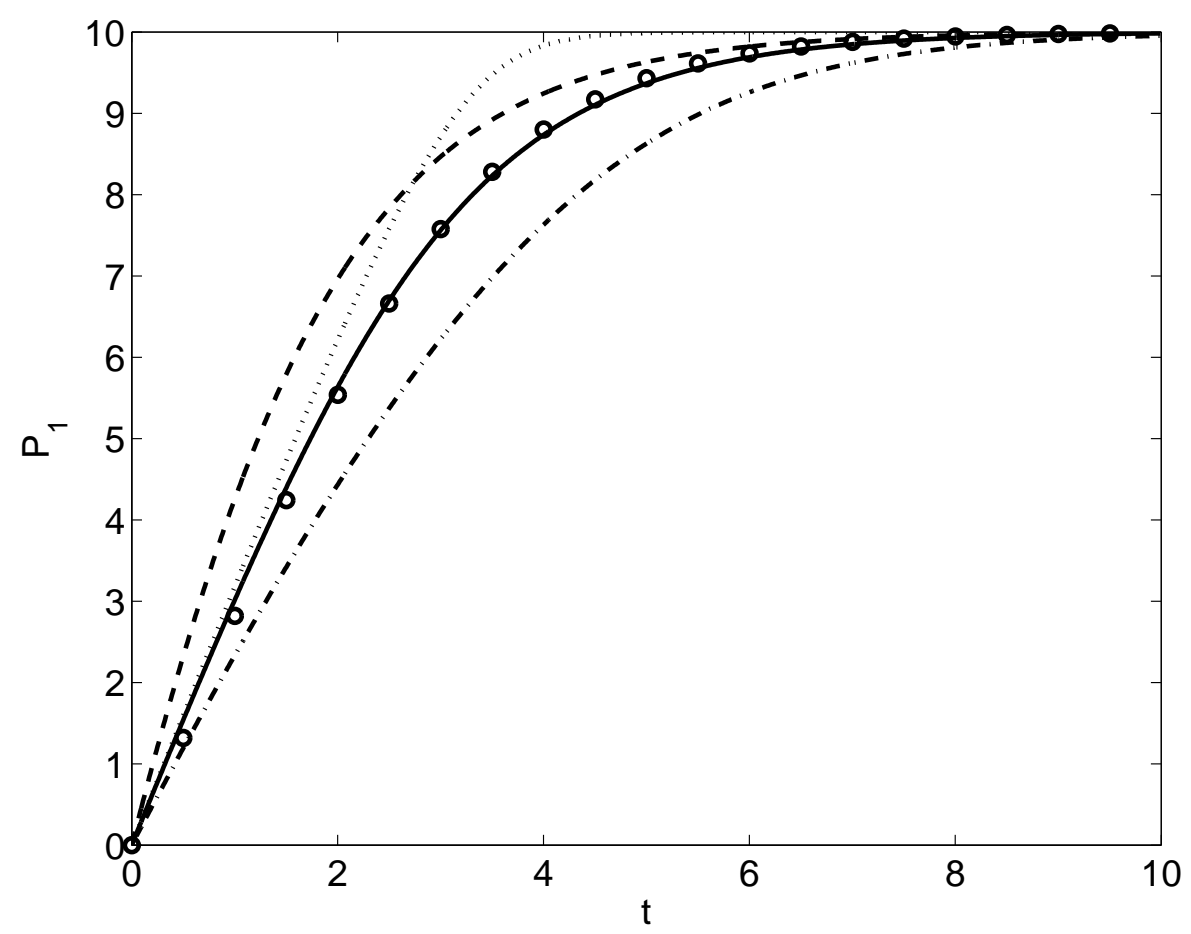

Figure 1. The tQSSA (full curve) approximates the full system (circles) very well, also when both the competitive SQSSA (dotted curve) and the single reaction tQSSA (dashed curve) do not. However, in this case we can not obtain that the competitive first order approximation (dash-dotted curve) is good. Parameters: $S_{1, T}=S_{2, T}=10, E_{T}=8, a_{1}=k_{1}=1, d_{1}=2, a_{2}=2, k_{2}=1, d_{2}=4\left(K_{1}^{M}=3\right.$, $\left.K_{2}^{M}=2.5, K=1, \epsilon=0.0137\right)$.

roughly valid if only $K \leq K_{M}$. This last assumption seems to be reasonable, since if $K_{1}^{M} \approx K_{2}^{M}$, we expect that the kinetic parameters $a_{i}$ and $k_{i}$ are similar for the two substrates. This would imply $K \approx k_{i} / a_{i}$ for the same $i$ and consequently $K \lesssim K_{M}$. Interestingly, for many metabolic enzymes $k \ll d$, i.e. $K \ll K_{M}$ (Atkinson, 1977). This implies that for competing substrates with identical affinities the relation $K \leq$ $K_{M}$ is even more reasonable, and even $K \ll K_{M}$ can be expected, in which case the tQSSA is a very good approximation, as seen from (36). Based on the above mentioned fact that often $k_{i} \ll d_{i}$, Bhalla and Iyengar (1999) use the relation $d_{i}=$ $4 k_{i}$. Then for identical affinities $a_{i}=5 k_{i} / K_{i}^{M}=5 k_{i} / K_{M}$, such that

$$
K=\frac{\max _{i} k_{i}}{\min _{i} a_{i}}=\frac{\max _{i} k_{i}}{\left(5 / K_{M}\right) \min _{i} k_{i}}=\frac{K_{M}}{5} \frac{\max _{i} k_{i}}{\min _{i} k_{i}}
$$



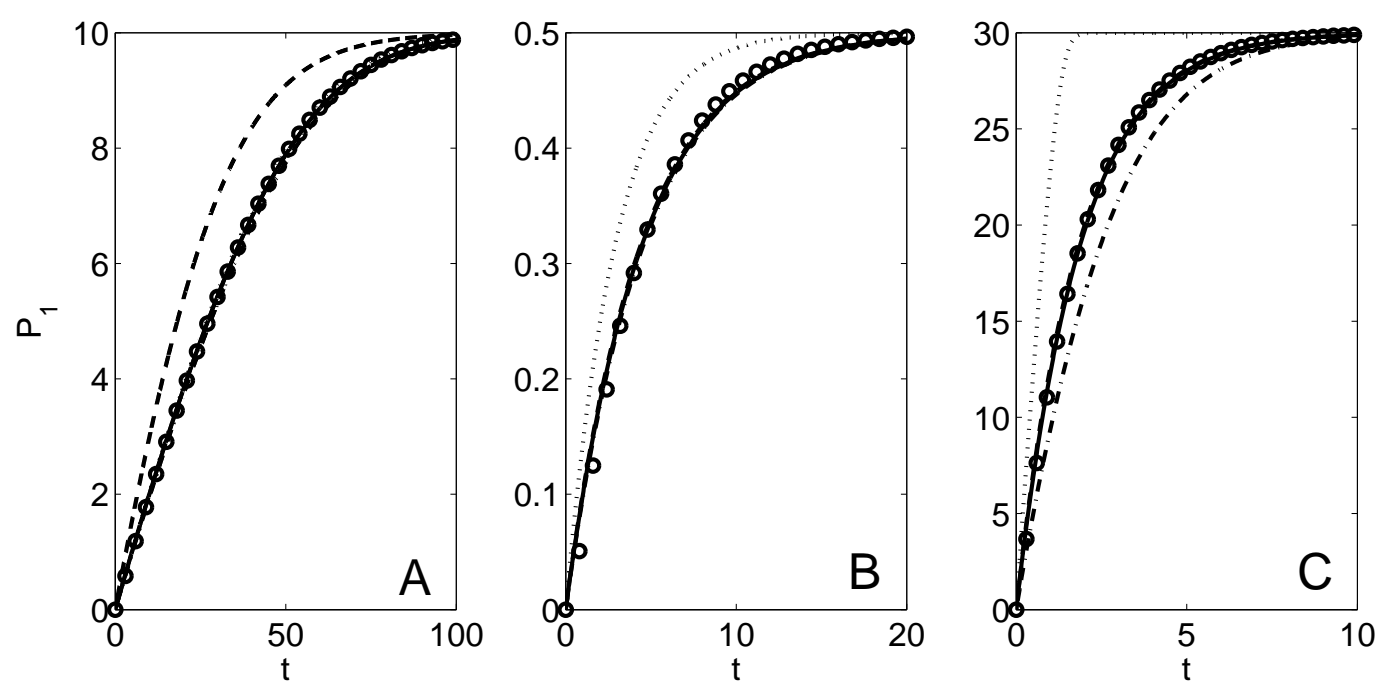

Figure 2. The first order approximation (dash-dot curve) coincides with the competitive SQSSA (dotted curve) when it is valid (panel A), and with the single reaction tQSSA (dashed curve) when the competition is negligible (panel B). However, at high enzyme concentrations the single reaction tQSSA is often a better approximation than the first order tQSSA (panel C). Parameters are $a_{1}=a_{2}=0.2, d_{1}=d_{2}=1, k_{1}=0.6, k_{2}=0.5$, $\left(K_{1}^{M}=8, K_{2}^{M}=7.5, K=3\right)$. In A: $S_{1, T}=S_{2, T}=10, E_{T}=1 . \epsilon=0.0038$. In B: $S_{1, T}=S_{2, T}=0.5, E_{T}=5 . \epsilon=0.0832$. In C: $S_{1, T}=S_{2, T}=30, E_{T}=100 . \epsilon=0.0219$.

from which it is seen that $K \leq K_{M}$ unless $k_{1}$ and $k_{2}$ differ by more than a factor 5. This is not the case for any of the IKK-2 data with $K_{1}^{M} \approx K_{2}^{M}$ from Kishore et al. (2003), nor for chitooligosaccharide with chitotriose and chitopentaose as substrates (Pi and Leary, 2004). In fact, their $k_{\text {cat }}$ (our $k_{i}$ ) values differ by less than a factor 2 .

The case $K_{1}^{M} \gg K_{2}^{M}$ was used to derive the first order approximation using a two-point Padé approximant. However, it is of its own biological interest as seen for example from the data by Pi and Leary (2004) for chitooligosaccharide with chitobiose as a substrate $\left(K_{1}^{M}=240 \mu \mathrm{M}\right)$ and, e.g., chitotriose as a competing substrate $\left(K_{2}^{M}=25 \mu \mathrm{M}\right)$. Fig. 3 shows that the the full tQSSA approximates the full system very well also in the case $K_{1}^{M} \gg K_{2}^{M}$, even when all other approximations fail. For $P_{1}$ (Fig. 3A) it is seen that the sQSSA overestimates the transient 

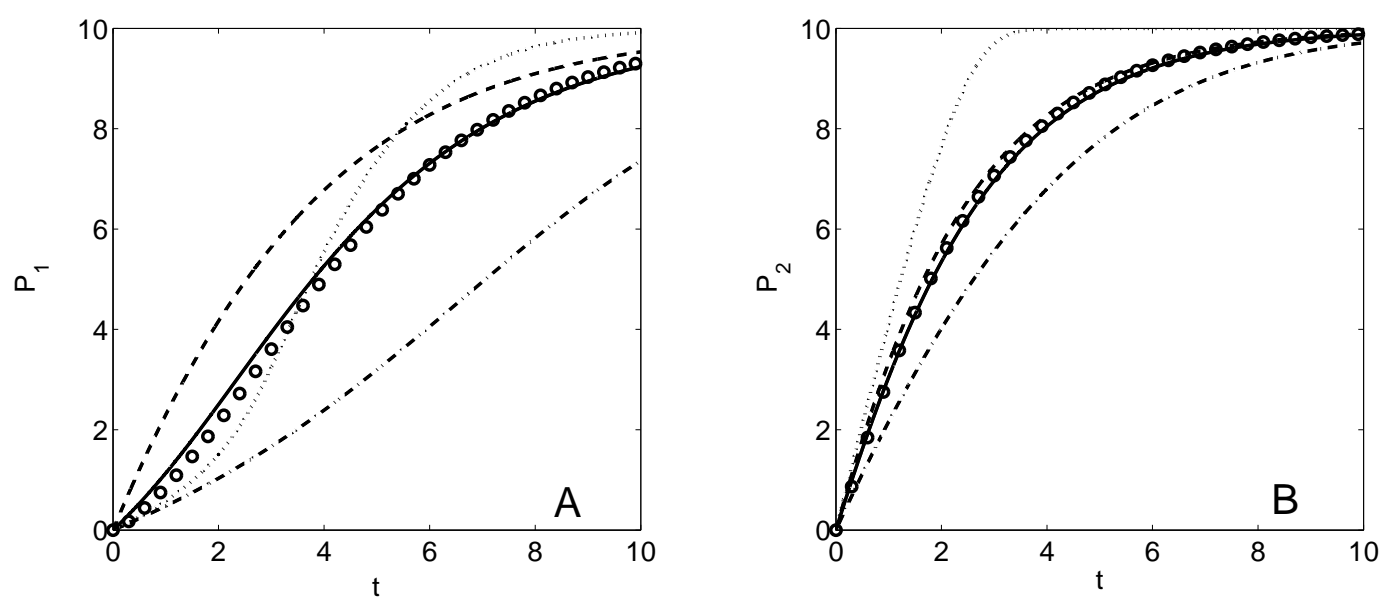

Figure 3. Also $K_{1}^{M} \gg K_{2}^{M}$ is captured well by the competitive tQSSA. Legends are as in Fig. 1, parameters are as in Fig. 2 except $a_{2}=2, S_{1, T}=S_{2, T}=E_{T}=10$. $\left(K_{1}^{M}=8, K_{2}^{M}=0.75, K=3\right) . \epsilon=0.0279$ ( $\bar{\epsilon}=0.0115$ from (33)).

phase in which mainly $P_{2}$ (Fig. 3B) is produced, where after it is accelerated, such that the overall behavior is not only quantitatively, but also qualitatively wrongly estimated in this example. Curiously, the single reaction tQSSA estimates $P_{2}$ well. The reason is the low degree of competition felt by the second reaction as seen from $\tilde{K}_{2, T}^{M} \approx 2 K_{2}^{M} \ll E_{T}$, so both $\tilde{K}_{2, T}^{M}$ and $K_{2}^{M}$ are negligible compared to $E_{T}$. This is not true for the first reaction as illustrated in Fig. 3A.

The assumption $K \leq K_{i}^{M}$ cannot be expected to hold when $K_{1}^{M} \gg K_{2}^{M}$, as illustrated by Fig. 3. Assuming again $d_{i}=4 k_{i}$, such that $a_{i}=5 k_{i} / K_{i}^{M}$, then gives

$$
K=\frac{\max _{i} k_{i}}{\min _{i} a_{i}}=\frac{\max _{i} k_{i}}{5 \min _{i} k_{i} / K_{i}^{M}} \leq \frac{K_{1}^{M}}{5} \frac{\max _{i} k_{i}}{\min _{i} k_{i}}
$$

so again $K \leq K_{1}^{M}$ unless $k_{1}$ and $k_{2}$ differ by more than a factor 5 . On the other hand

$$
K=\frac{\max _{i} k_{i}}{5 \min _{i} k_{i} / K_{i}^{M}} \geq \frac{\max _{i} k_{i}}{5\left(\max _{i} k_{i}\right)\left(\min _{i} 1 / K_{i}^{M}\right)}=\frac{K_{1}^{M}}{5},
$$

such that if $K_{1}^{M}>5 K_{2}^{M}$ we will have $K>K_{2}^{M}$. The parameters in Fig. 3 are such that $K_{1}^{M}>K>K_{2}^{M}$,

Related to the condition $K \leq K_{i}^{M}$ is the difference between $\epsilon$ from (32) and $\bar{\epsilon}$ from (33), which can be significant, as seen in Figs. 3 and 4. In Fig. 4 the (wrong) 


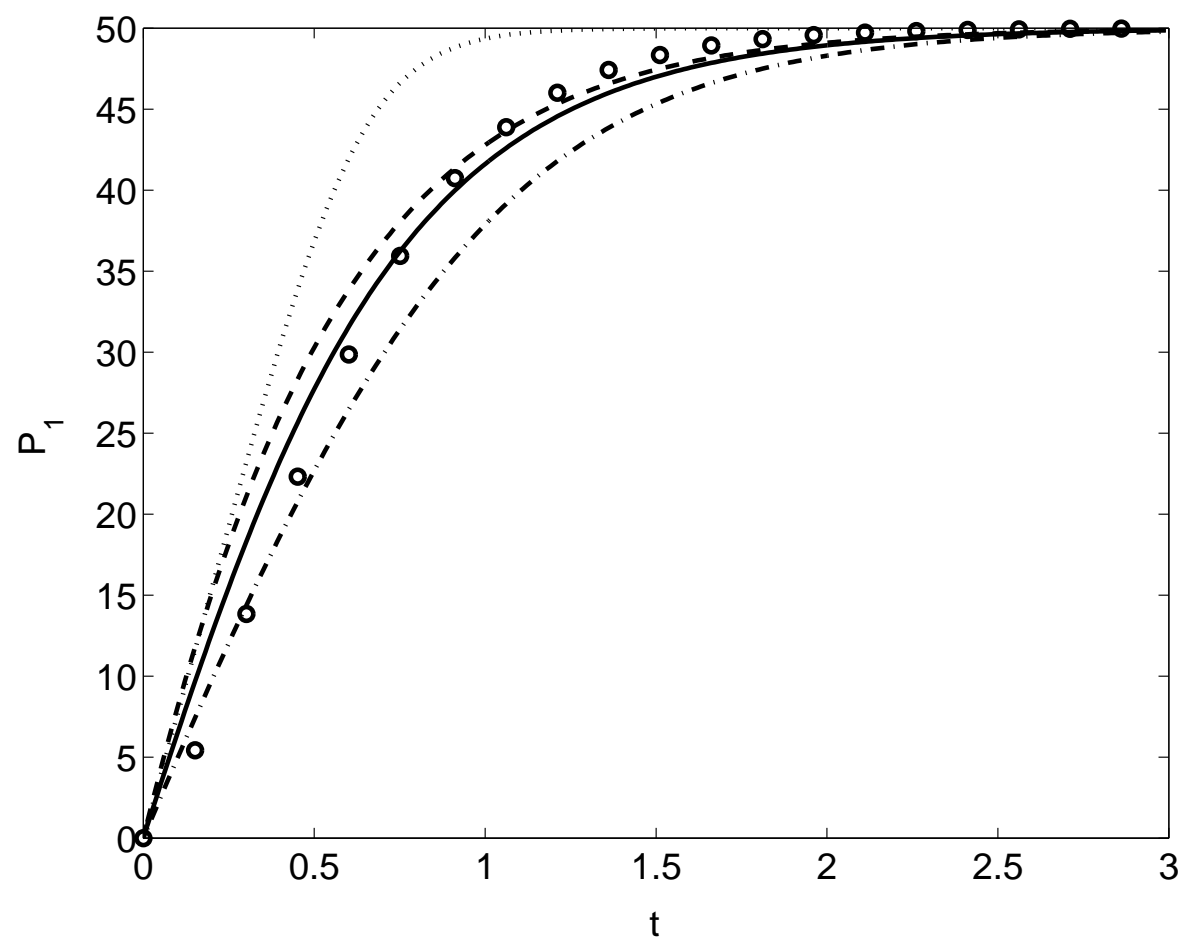

Figure 4. When $\epsilon$ becomes large also the tQSSA fails. Parameters are $S_{1, T}=S_{2, T}=50$, $E_{T}=70, a_{1}=0.1, k_{1}=2.99, d_{1}=0.01, a_{2}=0.2, k_{2}=4.99, d_{2}=0.1$ $\left(K_{1}^{M}=30, K_{2}^{M}=25.45, K=49.9\right)$ and $\epsilon=0.1494(\bar{\epsilon}=0.0829$ from (33)). expression from (33) gives $\bar{\epsilon}=0.0829$, which is very similar to $\epsilon$ in Fig. 2B where the tQSSA is a reasonable approximation. But in Fig. 4 the tQSSA does not fit well and indeed the correct formula from (32) gives a significantly higher parameter $\epsilon=0.1494$.

Our results are immediately applicable to, e.g., successive reactions catalyzed by the same enzyme, such as nonprocessive or distributive double phosphorylation or dephosphorylation processes, as seen for example in the MAPK cascade (Burack and Sturgill, 1997; Ferrell and Bhatt, 1997; Zhao and Zhang, 2001; Markevich et al., 2004). The reaction scheme can be seen as a special case of (2) with $P_{1}=S_{2}$ and is summarized as

$$
S_{1} \stackrel{E}{\longrightarrow} S_{2} \stackrel{E}{\longrightarrow} P
$$

where it is usually assumed that at the beginning only $S_{1}$ is present. Fig. 5 shows that the results presented here are often a good approximation. 

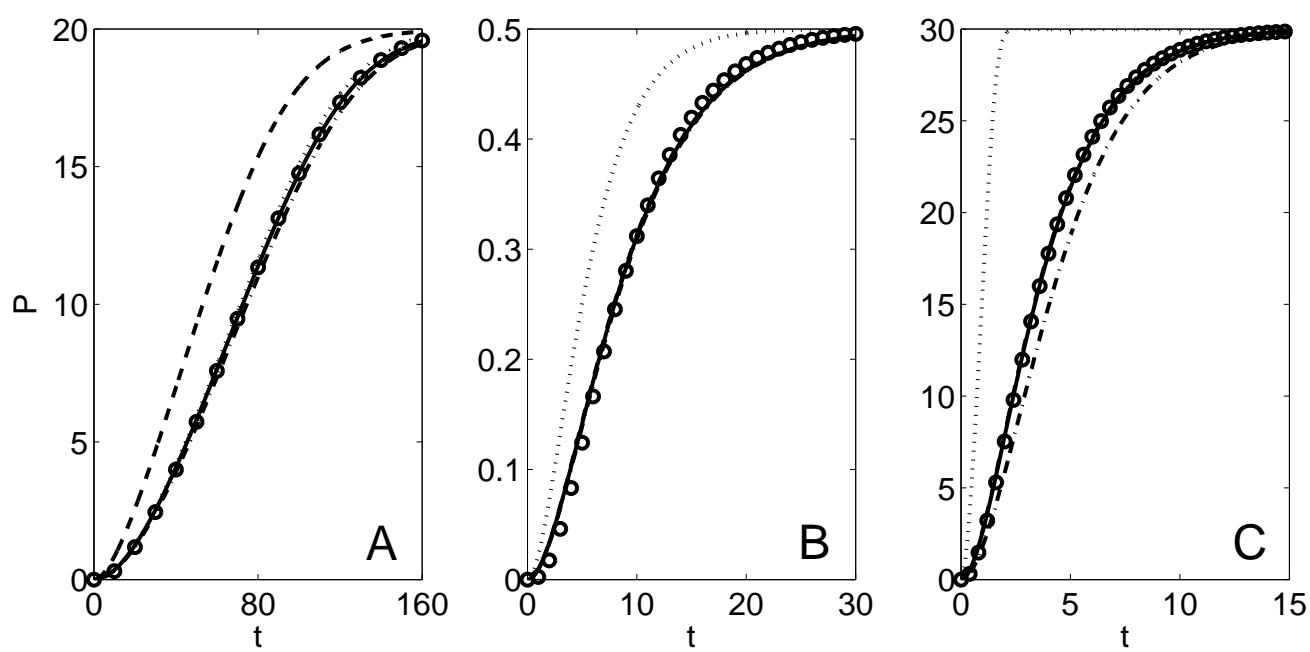

Figure 5. The tQSSA estimates well the development of the product of two successive reactions catalyzed by the same enzyme, and the discussion of the validity of the sQSSA, the single reaction tQSSA and the first order tQSSA apparently carries over to this case. Legends are as in Fig. 1, and parameters as in Fig. 2 except for the initial substrate concentrations, which are $S_{2, T}=0$ in all panels and: In A: $S_{1, T}=20$. In B: $S_{1, T}=0.5$. In C: $S_{1, T}=30$.

However, it should be remarked that our theoretical investigation of the validity of the tQSSA does not work in the case of successive reactions. The problem is that there is no $S_{2}$ at time $t=0$, and hence the time scales can not be found following Segel (1988) because the definition of the transient phase no longer holds. Nevertheless, it seems like the conclusions concerning the validity of the first order approximation from above carries over to this scenario (compare the three panels of Fig. 2 with the panels of Fig. 5).

We will present the investigation of such reactions in another paper.

Finding approximations extending the classical MM approach for complex reactions such as successive reactions, open systems, loops such as the GoldbeterKoshland switch (Goldbeter and Koshland, 1981), feedback systems etc. should be of great interest for further improving investigations and simulations of such reactions in vivo, where the MM description breaks down. The alternative is to sim- 
ulate each step of the reaction, i.e., the full system of ODEs, but for larger systems this can quickly become very computer expensive. Moreover, all of the (often unknown) kinetic parameters are needed for a full simulation, while a QSSA usually needs only $K_{M}$ and $V_{\max }$ values. Furthermore, the QSSA can provide theoretical insight which is hard to gain from the full system, for example in the way the MM approximation explains the saturation curve. We expect that the ideas presented here can be used to extend the tQSSA to the above (and, hopefully, other and more complex) reactions.

\section{A Validity of the first order approximation of the root of $\psi_{i}$}

To investigate the validity of (54) we evaluate $\psi_{1}$ from (24) at $C_{1}$ given by (53) This yields the remainder

$$
\begin{array}{r}
R_{1}:=\psi_{1}\left(C_{1}\right)=-E_{T}^{2} \bar{S}_{1}^{2} K_{2}^{M}\left[K_{2}^{M}\left(E_{T}+K_{1}^{M}\right)+K_{2}^{M} \bar{S}_{1}+K_{1}^{M} \bar{S}_{2}\right]^{-3} \times \\
{\left[K_{1}^{M} K_{2}^{M}\left(\bar{S}_{1} \bar{S}_{2}\left(K_{1}^{M}+K_{2}^{M}\right)+E_{T}\left(\bar{S}_{1} K_{2}^{M}+\bar{S}_{2} K_{1}^{M}\right)\right)+\right.} \\
\left.\bar{S}_{1}\left(K_{2}^{M}\right)^{3}\left(\bar{S}_{1}+K_{1}^{M}\right)+\bar{S}_{2}\left(K_{1}^{M}\right)^{3}\left(\bar{S}_{2}+K_{2}^{M}\right)\right]
\end{array}
$$

The term "remainder" is used, since if $R_{1}$ were zero, then $C_{1}$ given by (53) would be a true root, not only an approximation. To have a good approximation of the true root, $\left|R_{1}\right|$ must be small compared to typical sizes of $\psi_{1}$ such as

$$
\left|\psi_{1}(0)\right|=E_{T} K_{2}^{M} \bar{S}_{1}^{2} \quad \text { and } \quad \psi_{1}\left(\bar{S}_{1}\right)=\left(K_{1}^{M} \bar{S}_{1}\right)^{2}
$$

Similar conditions should hold for $C_{2}$ and $\psi_{2}$, but calculations and results are identical, and we show them only for $C_{1}$ in the following.

When (19) holds we expect (54) to hold, and then it reduces to the sQSSA (18). In this case the terms involving $E_{T}$ in $R_{1}$ are negligible and the condition $\left|R_{1}\right| \ll$ 
$\left|\psi_{1}(0)\right|$ implies

$$
\frac{E_{T}}{\left(\bar{S}_{1}+\tilde{K}_{1}^{M}\right)^{2}} \times \frac{K_{1}^{M}}{K_{2}^{M}}\left(\bar{S}_{2} \frac{K_{1}^{M}}{K_{2}^{M}}+\bar{S}_{1} \frac{\bar{S}_{2}+\tilde{K}_{2}^{M}}{\bar{S}_{1}+\tilde{K}_{1}^{M}}\right) \ll 1
$$

where we have introduced the so-called apparent MM constants (see, e.g., Schnell and Mendoza (2000))

$$
\tilde{K}_{i}^{M}=K_{i}^{M}\left(1+\bar{S}_{j} / K_{j}^{M}\right), \quad j \neq i .
$$

Similarly $\left|R_{1}\right| \ll \psi_{1}\left(S_{1}\right)$ can be restated as

$$
\left(\frac{E_{T}}{\bar{S}_{1}+\tilde{K}_{1}^{M}}\right)^{2}\left(\frac{\bar{S}_{2}}{K_{2}^{M}}+\frac{\bar{S}_{1}}{K_{1}^{M}} \frac{\bar{S}_{2}+\tilde{K}_{2}^{M}}{\bar{S}_{1}+\tilde{K}_{1}^{M}}\right) \ll 1 .
$$

These conditions are both clearly satisfied by (19) as long as $\bar{S}_{i}$ is not much greater than $K_{i}^{M}$, and $K_{1}^{M}$ and $K_{2}^{M}$ are of similar magnitude.

At high enzyme concentrations, (54) is a good approximation whenever $K_{1}^{M} \approx$ $K_{2}^{M}$, as stated in (41), which stimulates the assumption

$$
E_{T}+K_{i}^{M} \gg S_{1, T}+S_{2, T}, \quad i=1,2 .
$$

Our condition $\left|R_{1}\right| \ll\left|\psi_{1}(0)\right|$ then becomes

$$
\frac{E_{T}}{\left(E_{T}+\tilde{K}_{1}^{M}\right)^{2}} \times \frac{K_{1}^{M}}{K_{2}^{M}}\left(\bar{S}_{2} \frac{K_{1}^{M}}{K_{2}^{M}}+\bar{S}_{1} \frac{E_{T}+\tilde{K}_{2}^{M}}{E_{T}+\tilde{K}_{1}^{M}}\right) \ll 1,
$$

which is guaranteed by (A.5) if $K_{1}^{M}$ and $K_{2}^{M}$ are of similar magnitude.

The other condition, $\left|R_{1}\right| \ll \psi_{1}\left(S_{1}\right)$, is now

$$
\left(\frac{E_{T}}{E_{T}+\tilde{K}_{1}^{M}}\right)^{2}\left(\frac{\bar{S}_{2}}{K_{2}^{M}}+\frac{\bar{S}_{1}}{K_{1}^{M}} \frac{E_{T}+\tilde{K}_{2}^{M}}{E_{T}+\tilde{K}_{1}^{M}}\right) \ll 1 .
$$

This is on the other hand not guaranteed by (A.5); we must require, for example, that

$$
K_{i}^{M} \gg S_{i, T}
$$

Then (54) reduces to the single reaction first order tQSSA (14). 
At high enzyme concentrations, but low $\bar{S}_{i}$ and $K_{i}^{M}$ values, we can estimate the error that we make by using the first order tQSSA. The remainder $R_{1}$ from (A.1) is negative, which implies that (53) is an underestimate. The relative error $\operatorname{err}_{\text {rel }}$, given as the actual error $\mathrm{err} \leq \bar{S}_{1}-C_{1}$ divided by the maximal possible error $\bar{S}_{1}$, is then bounded by

$$
e r r_{\text {rel }} \leq \frac{\bar{S}_{1}-C_{1}}{\bar{S}_{1}}=\frac{K_{1}^{M}\left(1+\bar{S}_{2} / K_{2}^{M}\right)+\bar{S}_{1}}{K_{1}^{M}\left(1+\bar{S}_{2} / K_{2}^{M}\right)+\bar{S}_{1}+E_{T}} \leq \frac{\tilde{K}_{1, T}^{M}+S_{1, T}}{\tilde{K}_{1, T}^{M}+S_{1, T}+E_{T}}
$$

which is indeed small for large $E_{T}$, say

$$
E_{T} \gg \tilde{K}_{i, T}^{M}+S_{i, T}, \quad i=1,2
$$

Hence, only for an intermediate range of large, but not too large, values of $E_{T}$ is the first order approximation bad. When $K_{1}^{M} \approx K_{2}^{M}$, we can use (A.5) instead of (A.8) or (A.9) as a criterion for the first order approximation to be near the full tQSSA in agreement with (41).

\section{References}

Atkinson, D., 1977. Cellular Energy Metabolism and its Regulation. Academic Press, New York.

Baker, Jr., G. A., 1975. Essentials of Padé approximants. Academic Press, London.

Bhalla, U. S., Iyengar, R., 1999. Emergent properties of networks of biological signaling pathways. Science $283,381-387$.

Bisswanger, H., 2002. Enzyme Kinetics. Principles and Methods. Wiley-VCH.

Borghans, J., de Boer, R., Segel, L., 1996. Extending the quasi-steady state approximation by changing variables. Bull. Math. Biol. 58, 43-63.

Briggs, G. E., Haldane, J. B. S., 1925. A note on the kinetics of enzyme action. Biochem. J. 19, 338-339. 
Burack, W. R., Sturgill, T. W., 1997. The activating dual phosphorylation of MAPK by MEK is nonprocessive. Biochemistry 36, 5929-5933.

Ferrell, J. E., Bhatt, R. R., 1997. Mechanistic studies of the dual phosphorylation of mitogen-activated protein kinase. J. Biol. Chem. 272, 19008-19016.

Goldbeter, A., Koshland, Jr., D. E., 1981. An amplified sensitivity arising from covalent modification in biological systems. Proc. Natl. Acad. Sci. 78, 68406844.

Henri, V., 1901a. Recherches sur la loi de l'action de la sucrase. C. R. Hebd. Acad. Sci. $133,891-899$.

Henri, V., 1901b. Über das gesetz der wirkung des invertins. Z. Phys. Chem. 39, $194-216$.

Henri, V., 1902. Théorie générale de l'action de quelques diastases. C. R. Hebd. Acad. Sci. 135, 916-919.

Huang, C.-Y. F., Ferrell, J. E., 1996. Ultrasensitivity in the mitogen-activated protein kinase cascade. Proc. Natl. Acad. Sci. 93, 10078-10083.

Kholodenko, B. N., 2000. Negative feedback and ultrasensitivity can bring about oscillations in the mitogen-activated protein kinase cascades. Eur. J. Biochem. $267,1583-1588$.

Kishore, N., Sommers, C., Mathialagan, S., Guzova, J., Yao, M., Hauser, S., Huynh, K., Bonar, S., Mielke, C., Albee, L., Weier, R., Graneto, M., Hanau, C., Perry, T., Tripp, C. S., 2003. A selective IKK-2 inhibitor blocks NF- $\kappa$ B-dependent gene expression in interleukin-1 $\beta$-stimulated synovial fibroblasts. J. Biol. Chem. 278, $32861-32871$.

Markevich, N. I., Hoek, J. B., Kholodenko, B. N., 2004. Signaling switches and bistability arising from multisite phosphorylation in protein kinase cascades. J. Cell Biol. 164, 353-359.

Michaelis, L., Menten, M. L., 1913. Die kinetik der invertinwirkung. Biochem. Z. 49, 333-369. 
Pi, N., Leary, J. A., 2004. Determination of enzyme/substrate specificity constants using a multiple substrate ESI-MS assay. J. Am. Soc. Mass Spectrom. 15, $233-$ 243.

Rubinow, S., Lebowitz, J., 1970. Time-dependent Michaelis-Menten kinetics for an enzyme-substrate-inhibitor system. J. Am. Chem. Soc. 92, 3888-3893.

Schnell, S., Maini, P., 2000. Enzyme kinetics at high enzyme concentrations. Bull. Math. Biol. 62, 483-499.

Schnell, S., Maini, P., 2003. A century of enzyme kinetics: Reliability of the $k_{m}$ and $v_{\max }$ estimates. Comm. Theor. Biol. 8, 169-187.

Schnell, S., Mendoza, C., 1997. Closed form solution for time-dependent enzyme kinetics. J. Theor. Biol. 187, 207-212.

Schnell, S., Mendoza, C., 2000. Time-dependent closed form solutions for fully competitive enzyme reactions. Bull. Math. Biol. 62, 321-336.

Segel, L., 1988. On the validity of the steady state assumption of enzyme kinetics. Bull. Math. Biol. 50, 579-593.

Segel, L. A., Slemrod, M., 1989. The quasi steady-state assumption: a case study in pertubation. SIAM Rev. 31, 446-477.

Sols, A., Marco, R., 1970. Concentration of metabolites and binding sites. Implications in metabolic regulation. In: Current topics in Cellular Regulation. Vol. 2. Academic Press, New York.

Straus, O. H., Goldstein, A., 1943. Zone behavior of enzymes. J. Gen. Physiol. 26, $559-585$.

Tzafriri, A. R., 2003. Michaelis-Menten kinetics at high enzyme concentrations. Bull. Math. Biol. 65, 1111-1129.

Tzafriri, A. R., Edelman, E. R., 2004. The total quasi-steady-state approximation is valid for reversible enzyme kinetics. J. Theor. Biol. 226, 303-313.

Zhao, Y., Zhang, Z.-Y., 2001. The mechanism of dephosphorylation of extracellular signal-regulated kinase 2 by mitogen-activated protein kinase phosphatase 3 . J. 
Biol. Chem. 276, 32382-32391. 